\title{
Cyclic Cracking Resistance of Brittle Materials in Compressive Loading
}

\author{
A. G. Lanin
}

Scientific Institute "Luch," Podolsk, Russia

Fatigue behavior of brittle materials under compression is considered. The findings should be taken into account in the failure probability assessment of components made of materials with limited plasticity, which are used in various stress states.

Keywords: brittle material, compressive loading, fatigue behavior, cyclic cracking resistance.

Fatigue of brittle materials (interstitial phases, oxides, intermetallic compounds, graphite, etc.) produced mainly by powder metallurgy methods, has been examined far less extensively than that of metals. The absence of macroplastic deformation in powder metallurgy materials in a wide temperature range (in many cases, up to $0.5-0.8$ of the melting point [1-3]) limits the extent of manifestation of fatigue processes and stresses in the vicinity of the static strength [2]. In some experiments [3], the fatigue effect was not recorded at all (specimens of brittle materials either failed in the first load cycle or did not fail at all over a given test time); therefore, it appears that mechanical fatigue does not take place, and this impression is enhanced by the fact that in some materials (aluminum oxide [1], silicon nitride) fatigue failure is associated with stress corrosion.

At the same time, comparative tests on brittle materials under long-term static and cyclic loading, including tests in a highly corrosive atmosphere, e.g., tests on alumina, have revealed inherent fatigue processes caused by cyclic loading. It is evident that the process of fatigue failure of brittle materials results from the phenomenon of inelastic and highly localized deformation [2], and the intensity of this deformation governs the microcrack growth rate in the material.

In the majority of cases, the data on the crack growth rate in brittle materials were obtained in long-term loading of specimens of double cantilever type [2]. These data are represented in the form of a kinetic fatigue failure diagram in the coordinates of crack growth rate vs. stress intensity factor (SIF). The reports on fatigue behavior under other types of loading, e.g., under compressive loading which is encountered most often in structures made of powder metallurgy materials, are few in number.

The procedure of constructing a kinetic fatigue failure diagram (KFFD) is based on the method of determination of cracking resistance in uniaxial compressive loading, which is described in detail in [4].

The experimental data obtained in testing organic silicate glass, graphite, and zirconium carbide show that compression fracture is of kinetic nature [5]. The tests were conducted on flat specimens measuring $20 \times 30 \times 4 \mathrm{~mm}$ with a central straight initial crack of length $2 L(2 L=2-3 \mathrm{~mm})$, positioned at an angle $\beta$ to the compressive loading axis at a distance $d$ from each other. The growth and interaction of cracks in brittle solids under compressive stress state are shown 
(Fig. 1). From the fracture mechanics standpoint, this process consists of three conventional stages occurring successively with increasing compressive load. The first stage is represented by the equilibrium propagation of single cracks that initiate at defects in the material and do not interact with one another. The crack starts at a critical stress coefficient depending on its initial length and orientation. It follows a curvilinear trajectory approaching asymptotically the compressive loading axis. The minimum stress $\sigma$ required for the propagation of the most unfavorably oriented defects (at an angle of $30-45^{\circ}$ to the compressive loading axis) is 2.5-4 times higher than the stress $\sigma_{t}$ for loading of an initial crack perpendicular to the tensile loading axis. Subsequent damage accumulation is associated with the development of a system of interacting cracks, where the paired or multiple interactions between adjacent cracks (the intensity of these interactions increasing continuously) may lead to a qualitative change in the nature of their propagation, i.e., from equilibrium to unstable when the relative distance $\lambda=d / 2 L$ decreases to a critical value. The final stage of fracture becomes possible during the increased loading after certain multiple interactions of the cracks evolved in equilibrium, and at increased driving stress intensity with highly unstable crack growth. The load corresponding to fragmentation of the solid material is several times greater than the load at which the first macrocracks started to develop.

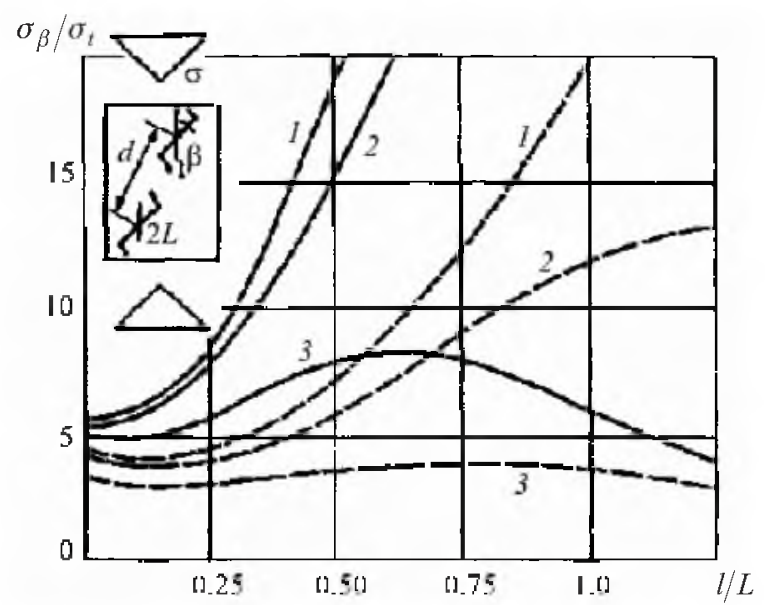

Fig. 1. Fracture diagrams for a system of parallel cracks: (I) $\lambda=\infty$; (2) $\lambda=3$; (3) $\lambda=2$ (solid lines $\beta=30^{\circ}$, dashed lines $\beta=45^{\circ}$ ).

Figure 2 shows schematically the sequence of tests performed in air at $20^{\circ} \mathrm{C}$ and the variation of crack growth conditions in a specimen under cyclic deformation. The specimen with an initial notch $l_{0}$ was statically loaded to a stress triggering the crack propagation from the notch tip. At that instant, the SIF reached the critical value $K_{I c}$ and, consequently, the crack "jumped" and then stopped as a result of SIF decreasing to $K_{\mathrm{I} a}$ which characterizes the crack inhibition condition in single-cycle loading. Subsequently, the specimen was subjected to cyclic deformation with a constant stress amplitude $\sigma_{c 0}$ at a stress ratio equal to zero and with a frequency of approximately $0.1 \mathrm{~Hz}$. This was accompanied by the fatigue crack growth with the rate slowing down due to the 
reduction of the SIF amplitude. For a non-zero cycle, the SIF amplitude was equal to the maximum SIF value of the cycle, i.e., $\Delta K_{\mathrm{I}}=K_{\mathrm{Imax}}=K_{\mathrm{I}}$.

When the fatigue crack growth rate reached approximately $10^{-3} \mathrm{~mm} /$ cycles (the condition of the acceptable test time), the testing at the stress amplitude $\sigma_{c 0}$ was interrupted and the specimen with the fatigue crack length $l$ was statically loaded to a stress $\sigma_{c i}$ whereby the SIF again reached $K_{\text {Ic }}$ and the crack that had completed the "jump" was arrested. Further cyclic deformation was carried out in the same manner with the stress amplitude $\sigma_{c i}$, and so on.

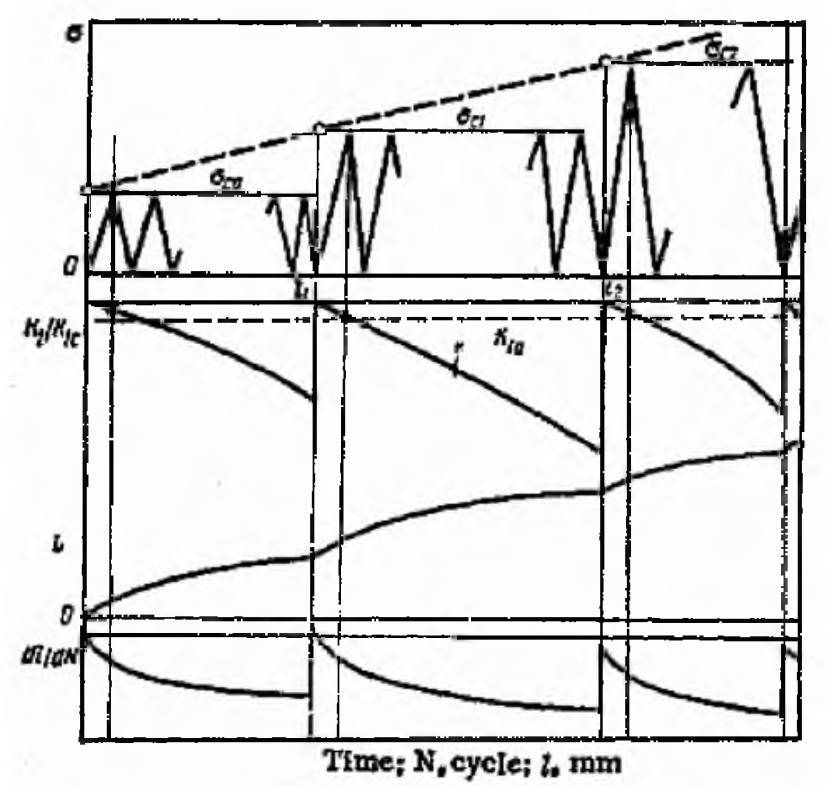

Fig. 2. Sequence of tests and variation of the crack growth conditions during cyclic loading with uniaxial compression.

To determine the SIF amplitude $K_{\mathrm{I}}=Y f(\sigma) \sqrt{l}$, the $K$-calibration of the $Y(l)$ function for each specimen was represented by a dashed line (the dashed line in Fig. 2) in the fracture diagram in the $\sigma$-vs.- $l / l_{0}$ coordinates. The inflection points of this line correspond to the experimentally determined critical stresses $\sigma_{c i}$ and crack length $l_{i}$ in the specimen under static loading. Instead of the absolute value of $K_{\mathrm{I}}$, we determined the ratio $K_{\mathrm{I}} / K_{\mathrm{Ic}}\left(l_{i j}\right)$.

The functional relationship between the crack growth rate and the SIF amplitude was set to be $d l / d N=a\left(K_{\mathrm{I}} / K_{\mathrm{I} c}\right)^{n}$, where $a$ and $n$ are the phenomenological parameters determined by the present method. Then, the equation was brought to the form $\ln (d l / d N)=\ln a+n \ln \left(K_{\mathrm{I}} / K_{\mathrm{Ic}}\right)$.

Analysis of test results in the high-amplitude region shows that the $\log (d l / d N)$ vs. $\log \left(K_{\mathrm{I}} / K_{\mathrm{Ic}}\right)$ functions exhibit a sharp inflection of $K_{\mathrm{I}}$ similar to $K_{\mathrm{Ic}}$ and approximating the arithmetic mean value of $K_{\mathrm{I} a}$. It is therefore useful to split the high-amplitude region of KFFD into two stages: (i) intensified crack growth at $K_{\mathrm{I}}<K_{\mathrm{I} a}$, and (ii) cyclic final fracture at $K_{\mathrm{I}}>K_{\mathrm{I} a}$. The parameters $n$ and $a$ for these two stages of the high-amplitude region, which are determined during the processing of experimental data, differ greatly. 
At the stage of cyclic final fracture, the value of parameter $n$ for all the materials, except for beryllium, is very high (up to 90) in comparison with $n=1-11$ for metals [10]. In fact, in the double logarithmic coordinates this section of KFFD degenerates into a vertical straight line: with increasing $K_{\mathrm{I}}$ the crack growth rate rapidly increases to a limiting value at $K_{\mathrm{I}}=K_{\mathrm{I} c}$. At the stage of intensified crack growth, the value of $n$ for all the materials, with the exception of alumina, steeply decreases and does not exceed 9.5. The parameter $a$ in transition from the stage of cyclic final fracture to the stage of intensified crack growth decreases as well. Thus, the parameters $n$ and $a$ greatly depend on the SIF amplitude.

A special feature of fatigue failure of ceramic materials is that their high defectiveness and structural heterogeneity together with the limited capacity for local stress relaxation result in noticeable time and geometrical nonuniformity of crack propagation. The crack grows by jumps due to processes of microplastic deformation after a specific number of cycles, which depends on $K_{\mathrm{I}}$. The fact that the KFFD of ceramic materials contains the stage of cyclic final fracture characterized by the high value of parameter $n$ causes the fatigue crack growth rate in the medium-amplitude region to extremely slow down (at $K_{\mathrm{I}} / K_{\mathrm{I} c}=0.5$ it is no higher than $2 \cdot 10^{-6} \mathrm{~mm} /$ cycles), Fig. 3, and be difficult to record experimentally even for the fairly ductile beryllium.

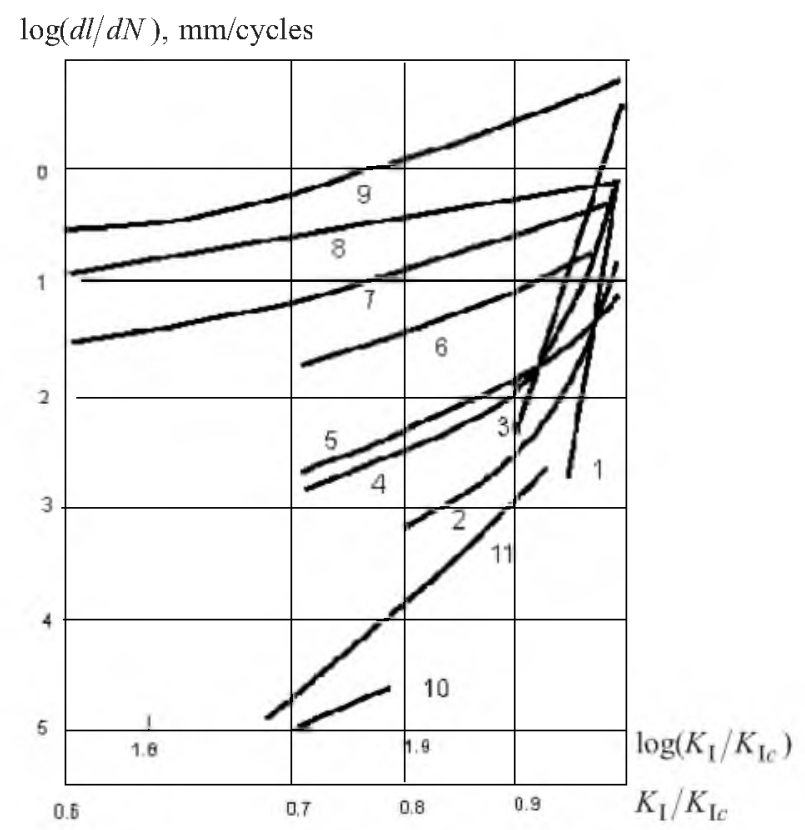

Fig. 3. Kinetic fatigue failure diagrams of structural materials for $K_{\mathrm{I}} / K_{\mathrm{Ic}}>0.6:(1) \mathrm{ZrC} ;(2,10) \mathrm{Be}$; (3) $\mathrm{Al}_{2} \mathrm{O}_{3}$; (4) GMR graphite; (5) ARV graphite; (6) ZrC + C; (7) aluminum 2024-T6; (8) steel 310; (9) steel $301 ;(11)$ graphite.

The quantitative agreement between the present findings and the data obtained on the WOL-type specimens and double cantilever beam indicates that the fracture mechanisms under compression, tension, and bending conditions are similar in nature. However, the crack propagation kinetics in cyclic compressive 
loading have a number of special features attributable mainly to the fact that the value of $K_{\mathrm{I}}$ decreases with increasing crack length. Consequently, the stress variation rate becomes comparable to the low rate of relaxation of local stresses; the probability of local deformation occurring and accumulating in the defective volume of a material increases, thus ensuring the equilibrium subcritical $\left(K_{\mathrm{I}}<K_{\mathrm{Ic}}\right)$ crack growth. The resultant residual tensile stresses during unloading add up with the stresses resulting from the applied load, and in subsequent loading cycles these residual stresses may also support subcritical crack growth If $\delta K_{\mathrm{I}} / \delta l>0$, the stress relaxation rate is considerably lower than the stress growth rate and the fatigue effect may not take place.

The results should be taken into account during the assessment of failure probability for components made of materials with limited plasticity, which are used in various stress states. It is evident that the method of cyclic compressive loading makes it possible to determine more accurately the relationships governing the fatigue failure of low-ductility materials in the case of changes of the structural parameters and chemical composition.

1. R. A. Andrievskii, A. G. Lanin, and G. A. Rymashevsky, Strength of Refractory Compounds [in Russian], Metallurgiya, Moscow (1974).

2. A. G. Lanin, Strength and Thermal Stress Resistance of Structural Ceramic [in Russian], Moscow State Engineering Physics Institute, Moscow (1998).

3. A. G. Lanin and I. I. Fedik, Thermal Stress Resistance of Materials [in Russian], Podolsk (2005).

4. A. G. Lanin, V. A. Sokolov, and N. A. Bochkov, "Determination of crack resistance of brittle materials," Strength Mater., 16, No. 2, 161-165 (1984).

5. A. G. Lanin, N. A. Bochkov, V. S. Egorov, and B. A. Sokolov, "Brittle fracture of materials in compression," Ibid, 17, No. 9, 1274-1281 (1986). 